\title{
Using Mobile Technology to Facilitate Reactive Case Detection of Malaria
}

\author{
Gordon Cressman*1, Michael McKay¹, Abdul-wahid Al-Mafazy², Mahdi M. Ramsan', \\ Abdullah S. Ali' ${ }^{2}$, Issa A. Garimo', Humphrey Mkali' ${ }^{2}$ and Jeremiah J. Ngondi ${ }^{1}$
}

${ }^{1}$ Information and Communication Technology, RTI International, Research Triangle Park, NC, USA; ${ }^{2}$ Zanzibar Malaria Elimination

Programme, Zanzibar Town, Tanzania, United Republic of

\section{Objective}

This presentation will share findings from more than three years of using mobile technology for reactive case detection (RACD) to help eliminate malaria in a well-defined geographic area. It will review the concepts of RACD, the application of mobile technology, lessons learned from more than three years of application, and considerations in applying this technology in other malaria elimination contexts.

\section{Introduction}

Zanzibar is comprised primarily of two large islands with a population of 1.3 million. Indoor Residual Spraying (IRS) campaigns, distribution of long-lasting insecticide treated bed nets (LLINs), and use of Rapid Diagnostic Tests (RDTs) have reduced Malaria prevalence from $39 \%$ in 2005 to less than $1 \%$ in $2011-2012$. As malaria burden decreases, there is an increasing need to track and follow up individual cases to contain transmission that could lead to resurgence. One method being used to achieve these aims is reactive case detection (RACD).

RACD is generally understood to be triggered whenever a case is identified by passive case detection. The response involves visiting the household of the newly reported case and screening family members. Depending on program protocol, it may also involve screening neighbors within a defined radius. RACD has been used or tested in Cambodia, China, India, Peru, Senegal, Swaziland, Tanzania, and Zambia. RACD can be resource intensive. Several studies raise questions concerning whether and how RACD can be prioritized and targeted effectively as case numbers continue to decline.

\section{Methods}

Since September 2012 Zanzibar Malaria Elimination Programme (ZAMEP) has used RACD to limit onward transmission, reduce the local parasite reservoir, and gather data needed improve program effectiveness. Zanzibar is one of very few malaria elimination contexts using a mobile technology system to support RACD. ${ }^{1}$ This system, called the Malaria Case Notification system (MCN) uses mobile software called Coconut Surveillance.

Coconut Surveillance is free and open source software designed for malaria elimination. It includes an interactive SMS system for case notification, a mobile software application designed to guide mobile case workers through RACD, and an analytics software application designed for surveillance and response program managers.

Data were collected in the Coconut Surveillance database for more than three years, beginning in September 2012. Reports were monitored in real time and periodically to assess RACD response times against protocol targets, case trends, case locations, and other data. Geographical Information System (GIS) software was used to produce detailed maps of case households. Three independent assessments were conducted of various aspects of the malaria surveillance system.

\section{Results}

From September 2012 to December 2015, Coconut Surveillance has helped malaria surveillance officers in Zanzibar respond to more than 8,617 (84\%) reported cases of malaria, complete nearly 10,245 household visits, test more than 36,185 household members, and identify and treat 2,032 previously unknown cases. The average number of RACD activities occurring within 48 hours increased from $72 \%$ in 2013 to $89 \%$ in 2015 . The number of household members screened during RACD also increased from 7,589 in 2013 to 14,987 in 2015. Challenges included incomplete registers at health care facilities, lack of transport, inadequate training for clinicians and surveillance officers, and insufficient communication to the affected communities.

\section{Conclusions}

In Zanzibar twenty malaria surveillance officers equipped with inexpensive Android tablets and motorbikes are keeping malaria prevalence at less than $1 \%$. The effectiveness of the system might be enhanced by improving training for clinicians and surveillance officers, ensuring the availability of transportation for surveillance officers, and improving communications to the affected communities. These results suggest key considerations for applying this and similar systems in other malaria elimination contexts.

\section{Keywords}

Malaria; Surveillance; Tanzania

\section{Acknowledgments}

RTI International and the Zanzibar Malaria Elimination Programme gratefully acknowledge the collaboration and support of the U.S. President's Malaria Initiative.

\section{References}

1. Ohrt C, Roberts KW, Sturrock HJ, Wegbreit J, Lee BY, Gosling RD Information Systems to Support Surveillance for Malaria Elimination. Am J Trop Med Hyg. 2015. doi: 10.4269/ajtmh.14-0257. PubMed PMID: 26013378.

\section{*Gordon Cressman}

E-mail: gmc@rti.org 